\title{
Ações de enfermagem na atenção ao portador de feridas na atenção básica em saúde
}

RESUMO | Objetivo: Descrever as ações do enfermeiro no atendimento ao portador de feridas na atenção básica em saúde. Método: Revisão integrativa estruturada em seis etapas a partir da busca de artigos de livre acesso nas bases de dados publicados no intervalo de 10 anos (2009-2019), baseado em critérios de elegibilidade estabelecidos e no nível de evidência das publicações. Resultados: Os estudos incluídos para análise apontam a atuação do enfermeiro centrada na garantia de espaço físico em conformidade com as normas sanitárias, na educação em saúde como ação de promoção da autonomia e autocuidado e na observação dos aspectos sociais e culturais do usuário no contexto da melhoria da cicatrização das lesões. Conclusões: Entende-se como necessária a inserção deste profissional enquanto participante ativo da assistência para desenvolvimento de ações respaldadas e na possibilidade da redução do tempo de tratamento do usuário e dos custos da assistência junto ao sistema de saúde.

Palavras-chaves: Ferimentos e lesões; Atenção primária à saúde; Cuidados de enfermagem; Enfermagem.

\begin{abstract}
Objective: To describe the actions of nurses in the care of patients with wounds in primary health care. Method: Integrative review structured in six stages based on the search for freely accessible articles in the databases published over a 10year period (2009-2019), based on established eligibility criteria and the level of evidence of the publications. Results: The studies included for analysis point out the role of nurses focused on guaranteeing physical space in accordance with health standards, in health education as an action to promote autonomy and self-care and in observing the social and cultural aspects of the user in the context of improvement of wound healing. Conclusions: It is understood as necessary to insert this professional as an active participant in the assistance for the development of supported actions and in the possibility of reducing the user's treatment time and the costs of assistance with the health system.
\end{abstract}

Keywords: Wounds and injuries; Primary health care; Nursing care; Nursing.

RESUMEN I Objetivo: Describir las acciones del enfermero en la atención de pacientes con heridas en la atención primaria de salud. Método: Revisión integradora estructurada en seis etapas a partir de la búsqueda de artículos de libre acceso en las bases de datos publicadas durante un período de 10 años (2009-2019), en base a los criterios de elegibilidad establecidos y al nivel de evidencia de las publicaciones. Resultados: Los estudios incluidos para análisis señalan el rol del enfermero enfocado en garantizar el espacio físico de acuerdo con los estándares de salud, en la educación para la salud como una acción para promover la autonomía y el autocuidado y en la observación de los aspectos sociales y culturales del usuario en el contexto de mejora de la cicatrización de heridas. Conclusiones: Se entiende necesario insertar a este profesional como participante activo en la asistencia para el desarrollo de acciones apoyadas y en la posibilidad de reducir el tiempo de tratamiento del usuario y los costos de asistencia con el sistema de salud.

Palabras claves: Heridas y lesiones; Primeros auxilios; Cuidado de enfermera; Enfermería.

\section{Millena Rebeca Pereira de Oliveira}

Enfermeira. Centro Universitário Tabosa de Almeida (ASCES-UNITA).

ORCID: 0000-0003-1830-9316

\section{Luan José Queiroz de Lima}

Enfermeiro. Centro Universitário Tabosa de Almeida (ASCES-UNITA).

ORCID: 0000-0001-8338-6436

\section{Carla Rayane Santos Dutra}

Enfermeira. Centro Universitário Tabosa de Almeida (ASCES-UNITA).

ORCID: 0000-0003-0321-5560

\section{Maria Eduarda Santos Silva}

Enfermeira. Centro Universitário Tabosa de Almeida (ASCES-UNITA).

ORCID: 0000-0002-4020-6981
Maria Eduarda dos Santos

Graduanda em Enfermagem. Centro Universitário Tabosa de Almeida (ASCES-UNITA). ORCID: 0000-0003-1699-7266

\section{Ester Pereira Silva}

Graduanda em Enfermagem. Centro Universitário Tabosa de Almeida (ASCES-UNITA). ORCID: 0000-0002-7735-5526

\section{Diego Augusto Lopes Oliveira}

Enfermeiro. Doutorando em Enfermagem do PPGENF-UFPE. Docente do curso de graduação em enfermagem da ASCES-UNITA. ORCID: 0000-0003-1754-7275

Recebido em: 26/01/2021

Aprovado em: 09/02/2021
INTRODUÇÃO

o âmbito da saúde pública a
assistência a portadores de le-
sões é uma problemática frequente. Apesar da crescente demanda na maioria dos serviços de saúde do país, os registros referentes ao atendimento dessa clientela são escassos, assim como os estudos sobre o impacto reproduzidos por esses agravos. $\mathrm{O}$ atendimento ao cliente portador de lesões necessita de atenção especial por parte dos profissionais de saúde, em especial, do enfermeiro devido às suas habilidades técnicas para realização da avaliação da lesão e implementação do tratamento através do uso do curativo ideal. ${ }^{(1)}$

O gerenciamento da sala de curativo e da utilização de insumos no tratamento 
é outra atribuição de grande importância que demanda conhecimento científico para a sua prática. Pode-se destacar ainda, a realização de ações relacionadas à promoção e educação em saúde da comunidade, envolvendo tecnologias e recursos acessíveis que possibilitem o rastreamento, identificação e intervenção precoce no tocante às lesões de pele, definidas através de regulamentações profissionais associadas ao exercício legal da enfermagem $^{(2)}$. Essas atividades devem objetivar o estreitamento da relação com a equipe da unidade básica de saúde, o conhecimento para autonomia e promoção do autocuidado bem como desmistificar as relações culturais e sociais que interferem no contexto do cuidar de feridas. ${ }^{(1)}$

Estudos e pesquisas nessa temática que abordam a questão do cuidado com feridas, em sua maioria, revelam uma preocupação com a objetividade, adotando uma perspectiva curativista cujo foco é o tratamento da ferida com pouca ênfase na questão educativa voltada para a pessoa com feridas e para seus conhecimentos e experiências no tratamento e prevenção das mesmas ${ }^{(3)}$.

As ações desenvolvidas pelo enfermeiro nesse nível assistencial devem abordar o usuário de forma integral e individualizada, sendo capaz de viabilizar além da cicatrização ou cura da lesão a melhora clínica e social do mesmo, através de procedimentos eficientes e otimização do atendimento, assegurando o direito dos usuários à assistência de qualidade e equânime. Na gestão e/ou na execução das práticas assistenciais, educativas e preventivas, no nível da atenção básica, o trabalho do enfermeiro é estratégico e indispensável, sendo assegurada sua inserção nas equipes e nos territórios por meio dos marcos programáticos e legais do Sistema Único de Saúde (SUS). ${ }^{(4)}$

Compreende-se que o cenário da atenção básica em saúde possui alta relevância no cuidado prestado pela equipe de enfermagem ao cliente com lesões por se configurar como a principal porta de entrada da comunidade aos níveis as- sistenciais dispostos no âmbito do SUS. A fragilidade de políticas públicas específicas ao portador de lesões que norteiam a prática do profissional de enfermagem e a carência de estudos que evidenciam essa temática se colocam como plano de fundo da problemática enredo deste estu$\mathrm{do}^{(4)}$. O presente artigo tem como objetivo elucidar as ações do enfermeiro no atendimento ao portador de feridas na atenção básica em saúde.

\section{MÉTODO}

Trata-se de um estudo de revisão integrativa da literatura que se caracteriza por incorporar informações e ideias que possibilitam fortalecimento da prática baseada em evidências (PBE)(5). Para desenvolvimento do estudo elencou-se enquanto pergunta norteadora: "Quais as ações desenvolvidas pelo enfermeiro no cuidado ao portador de feridas na atenção básica em saúde?". A partir desta desencadearam-se as etapas relacionadas a seleção de materiais que possibilitaram argumentação acerca desta temática baseadas no protocolo de construção de estudos de revisão PRISMA (Preferred Reporting items for Systematics Reviews and Meta-analyses).

Nesse sentido, identificaram-se as bases de dados do LILACS, MEDLINE e BDENF como sítios para busca através do uso dos descritores (DECS-BIREME): ferimentos e lesões/ wounds and injuries/ Heridas y traumatismos; cuidados de enfermagem/ Nursing care/ Atención de enfermería; Atenção primária à saúde/ Primary health care/ Atención primaria de Salud, ligados a partir da conexão booleana AND.

Consideraram-se como critérios para busca estudos de livre acesso, publicados no intervalo de 10 anos (2009-2019), com texto na íntegra e nos idiomas português, inglês e espanhol. Foram eliminados os estudos provenientes de trabalhos de especialização, dissertações e teses; informativos de programas de saúde específicos da área ou relacionados a temática. Estudos que não apresentem relação com a temática da assistência a feridas na aten- 
ção básica em saúde e estudos de revisão integrativa da literatura. A busca nas bases de dados ocorreu nos meses de janeiro e fevereiro de 2020.

Após a seleção dos artigos nas bases foi estabelecido processo de análise dos dados, considerando avaliação por pares, através da leitura sistemática do título, do resumo e do texto completo, sendo eliminados da amostra de análise desta revisão os que não apresentassem coerência com a pergunta norteadora e objetivo de pesquisa ${ }^{(4)}$. Um outro aspecto elucidado no processo de análise dos dados, em coerência com a PBE, é o estabelecimento da classificação das publicações analisadas a partir do nível de evidência. A classificação das evidencias dos estudos considerou a hierarquia de evidências para estudos de intervenção, sendo: Nível I - revisão sistemática ou metanálise; Nível II - estudos controlados e aleatórios; Nível III - estudos controlados sem randomização; Nível IV - estudos caso-controle ou de coorte; Nível V - revisão sistemática de estudos qualitativos ou descritivos; Nível VI - estudos qualitativos ou descritivos e Nível VII - opiniões ou consensos. ${ }^{(6)}$

\section{RESULTADOS}

Mediante a aplicação dos critérios estabelecidos para busca nas bases de dados obtiveram-se 79 estudos (MEDLINE (61); LILACS (9); BDENF (9)) sob os quais desenvolveu-se a etapa de identificação que deu início ao processo de análise dos

\section{Figura 1 - Fluxograma representativo da busca de artigos. Caruaru - PE, Brasil, 2020.}

"Quais os ações desenvolvidas pelo enfermeiro no cuidado ao portadores no atenção básica em saúde?"

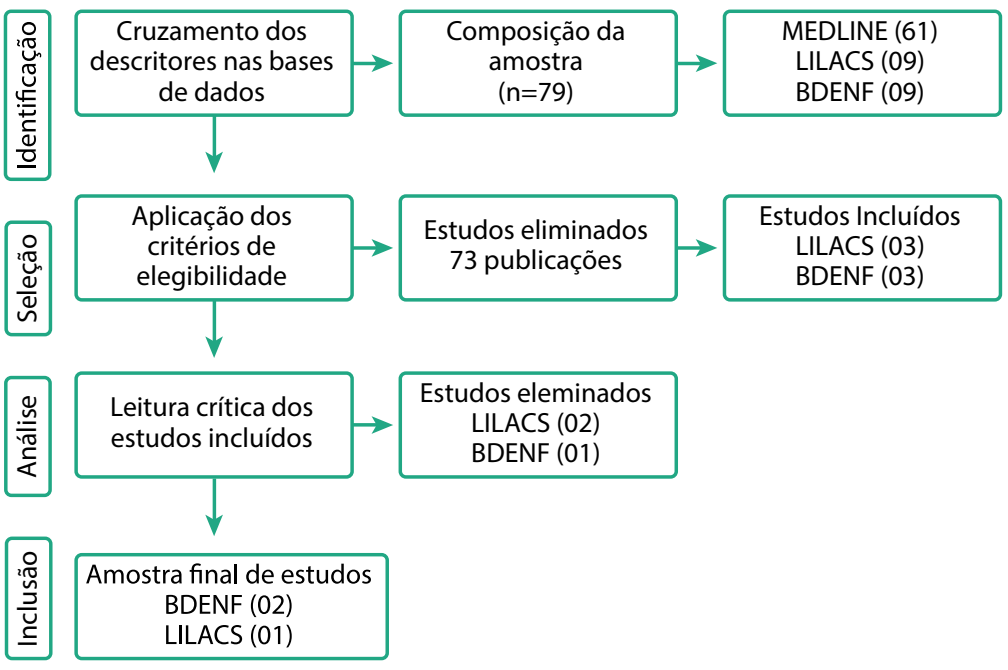

dados nas etapas estabelecidas, conforme explicitado na figura 1:

$\mathrm{Na}$ etapa de seleção foram aplicados os critérios de elegibilidade estabelecidos na pesquisa através dos quais foram eliminados 73 estudos: (02) - idioma do estudo: sueco e alemão; (51) - Ano de publicação diferente do intervalo estabelecido; (05) Acesso restrito/pago; (04) - Sem relação com o tema; (02) - Material informativo; (01) - Tese; (08) - Duplicados nas bases de dados. Nesse sentido restaram 06 estudos para leitura crítica e análise dos dados. $\mathrm{Na}$ etapa de análise foi procedida a leitura do material (título, resumo e texto completo) possibilitando a eliminação de 03 estudos (02 na etapa da leitura do título e 01 na leitura do resumo), restando 03 estudos incluídos para análise do texto completo. Os estudos incluídos nesta etapa estão identificados no quadro 1 :

Mediante a análise detalhada dos estudos incluídos para etapa final de análise destacaram-se os pontos de maior relevância na produção e seu alinhamento no tocante ao direcionamento proposto pela pergunta norteadora deste estudo e formulou-se quadro com o detalhamento destas produções.

\section{DISCUSSÃO}

A assistência ao portador de feridas é um processo dinâmico e individualizado e a competência demonstrada pelo enfermeiro através de suas habilidades são imprescindíveis para garantia e manutenção da qualidade durante todo decorrer do cuidado. Tal perspectiva é demonstrada a partir da aplicação dos conhecimentos da semiologia e semiotécnica que permitem uma avaliação integral e a indicação dos

Quadro 1 - Relação dos artigos inclúidos de acordo com as variáveis do estudo. Caruaru - PE, Brasil, 2020.

\begin{tabular}{|c|c|c|c|c|}
\hline ID & AUTORES & TíTULO DA PUBLICAÇÃO & ANO & PERIÓDICO \\
\hline 01 & Aguiar, JS et. al. ${ }^{(7)}$ & Estrutura física e recursos materiais das salas de curativos das policlínicas regionais. & 2019 & Reuol UFPE \\
\hline 03 & Chibante, CLP et. al. ${ }^{(9)}$ & Saberes e práticas no cuidado centrado na pessoa com feridas & 2017 & Esc. Anna Nery \\
\hline
\end{tabular}


Quadro 2 - Detalhamento dos artigos incluídos para análise de texto completo. Caruaru - PE, Brasil, 2020.

\begin{tabular}{|c|c|c|c|c|c|}
\hline ID & OBJETIVO & MÉTODO & RESULTADOS & CONCLUSÕES & $\begin{array}{l}\text { NÍVEL DE } \\
\text { EVIDÊNCIA }\end{array}$ \\
\hline 01 & $\begin{array}{l}\text { Identificar a estrutura } \\
\text { física e materiais das } \\
\text { salas de curativos de } \\
\text { policlínicas regionais } \\
\text { para atendimento ao } \\
\text { cliente com feridas. }\end{array}$ & $\begin{array}{l}\text { Estudo qualitativo, } \\
\text { observacional e } \\
\text { transversal. }\end{array}$ & $\begin{array}{l}\text { Foram identificadas as lacunas relaciona- } \\
\text { das à estrutura física e recursos materiais } \\
\text { através da aplicação de dois checklists, } \\
\text { esses permitiram visualizar a adequação } \\
\text { das salas de curativo, disponibilidade de } \\
\text { insumos e as implicações que estas traziam } \\
\text { na manutenção do cuidado ao portador de } \\
\text { feridas. A atuação do Enfermeiro é neces- } \\
\text { sária pela condição de liderança e de co- } \\
\text { nhecimento acerca dos dispositivos legais, } \\
\text { normas sanitárias e materiais necessários } \\
\text { para manutenção e perfeito funcionamento } \\
\text { da sala de curativos. }\end{array}$ & $\begin{array}{l}\text { O estudo destaca a inexistência } \\
\text { de uma unidade ideal para o } \\
\text { atendimento ao portador de } \\
\text { feridas, enfatizando a ausência } \\
\text { de estrutura física e materiais } \\
\text { adequados diante da necessida- } \\
\text { de apresentada pela população } \\
\text { e os eventuais riscos que estas } \\
\text { lacunas provocam aos usuários } \\
\text { e profissionais. }\end{array}$ & VI \\
\hline
\end{tabular}

tratamentos adequados as necessidades do usuário ${ }^{(10-11)}$.

Para que esta assistência seja efetiva o Ministério da Saúde (MS) preconiza que os serviços ofereçam espaço específico com estrutura física apropriada, produtos e materiais que atendam as necessidades biopsicossociais dos usuários. O atendimento deve ser realizado em ambiente com dimensões adequadas, disponibilidade de produtos e coberturas específicas para cada tipo de ferida e que considere a individualidade de cada paciente. Estudos enfatizam a indisponibilidade de ambientes adequados para atendimento ao portador de feridas, sendo esta ocasionada por problemas relacionados a manutenção da estrutura física e desfalque 
de insumos (materiais e coberturas especiais) que comprometem a continuidade do tratamento e expõem a população a eventuais riscos provocados por estas lacunas. Neste panorama emerge a necessidade da intervenção do enfermeiro, por este dominar o conhecimento acerca das normas sanitárias específicas para o atendimento ao portador de feridas e conhecer os insumos necessários para a prestação do cuidado diante dos casos de maior prevalência de atendimento. ${ }^{(12-13,14)}$

O modelo assistencial desenvolvido pelo Enfermeiro requer que os saberes sejam compartilhados através de estratégias que incluam a família, auxiliando a compreender o indivíduo integralmente e visando promover medidas de bem-estar e qualidade de vida. A Consulta de Enfermagem promove ambiente de compartilhamento de conhecimentos (científico e popular), provocando mudanças de comportamento através da inserção de práticas de saúde aceitas pelo usuário e que promovam impacto no processo de cicatrização. Essa estratégia possibilita a quebra do modelo biológico-visível e respeita o contexto biopsicossocial, es- piritual e cultural do indivíduo, família e comunidade, inserindo-os como protagonistas do processo de cuidado. ${ }^{(15-16,17)}$

A ausência de intervenções do enfermeiro, a não inserção do paciente enquanto protagonista do processo de cuidado e a não consideração das suas variáveis culturais, são fatores que levam ao distanciamento do usuário da unidade básica de saúde, não adesão e/ou abandono do tratamento proposto. A falta de um diálogo sobre a ausência nos dias de procedimento, entender os motivos e buscar soluções juntos são iniciativas esperadas de um profissional de enfermagem comprometido com a saúde.

A temática ferida remete a diversos problemas de ordem de saúde pública no Brasil, especialmente pelas complicações e alto custo envolvidas no seu gerenciamento e tratamento. Estudos apontam que pessoas portadoras de feridas podem desenvolver algum tipo de complicação de ordem física ou emocional ao longo da vida e que a educação em saúde promovida pelos enfermeiros é algo essencial, pois o diálogo e a interação são as principais formas de construção de conhecimentos e empoderamento dos usuários e familiares para o desenvolvimento da autonomia no cuidado e promoção à saúde. ${ }^{(18-19)}$

No entanto é de extrema importância que o paciente seja protagonista nesse cuidado tendo sua autonomia preservada e incentivada, contribuindo no processo de recuperação e cicatrização da ferida. O enfermeiro deve respaldar a autonomia e exercer papel de acompanhamento e orientação das práticas desenvolvidas pelo usuário e família através do uso da tecnologia de educação em saúde apropriada para o desenvolvimento da autonomia do usuário de forma contínua e flexível, tendo assim a oportunidade de evoluir na oferta de um atendimento de qualidade ao usuário. ${ }^{(20)}$

Entende-se, mediante ao levantamento teórico estabelecido, a necessidade de elencar os aspectos relevantes e de impacto nas ações do profissional enfermeiro na atenção ao portador de lesões no cenário da atenção básica em saúde, sendo estes descritos no quadro 3:

Observou-se enquanto limitação na produção deste estudo a baixa disponi-

\section{Quadro 3 - Principais ações do enfermeiro na assistência ao portador de lesões na atenção básica em saúde. Caruaru - PE, Brasil, 2020.}

Acolher o usuário considerando seu histórico de saúde pregresso, cultura, raça, sexo, determinantes sociais e contexto familiar.

Garantir ambiente (sala de curativos) adequado às normas sanitárias para acomodação do usuário e realização do procedimento livre de riscos e danos a sua saúde.

Realizar consulta de enfermagem e executar exame físico completo e específico (área da lesão) como meio de estabelecer avaliação completa do usuário.

Indicar tratamento adequado às características físicas gerais do usuário e específicas da lesão.

Prover insumos para realização dos procedimentos garantindo sua utilização dentro das necessidades do usuário e que não aumente os custos de forma a gerar ônus aos serviços de saúde.

Oportunizar atividades de educação em saúde para promoção das mudanças de hábitos e consequente adesão a um estilo de vida que possibilite aumento no panorama de cicatrização da lesão.

Realizar visita domiciliar como meio de oportunizar educação em saúde ao usuário e família para reflexão quanto aos hábitos de vida e desmistificar os dogmas culturais envolvidos no processo de cuidar

Manter registro das intervenções de enfermagem (escrito e fotográfico) para avaliação do resultado das ações sistematizadas do cuidado implementadas.

Oportunizar momentos de discussão dos casos acompanhados na comunidade entre os profissionais de saúde componentes da equipe de saúde da família (Médico, Agentes comunitários de Saúde e Técnicos de Enfermagem) para fortalecimento das ações de cuidado ao portador de lesões.

Desenvolver protocolos de cuidado aos portadores de lesão na comunidade de forma a estabelecer linha de cuidado específica e que privilegie o usuário dentro de suas especificidades em saúde.

Fonte: Autores, 2019 
bilidade de fontes atualizadas que tratem da temática nesta modalidade de atenção. Em contrapartida, o estudo contribui no estímulo aos leitores, instituições e profissionais de saúde quanto a necessidade de desenvolver ações visando a capacitação das equipes de enfermagem para intervenção junto aos usuários no sentido de aumentar as evidências científicas no cuidado, bem como, na utilização dos insumos e coberturas de forma adequada e sem gerar custos adicionais ao sistema de saúde.

\section{CONCLUSÃO}

Pela observação dos aspectos analisados entende-se que as ações de enfermagem direcionadas ao portador de lesões no cenário da atenção básica em saúde estão relacionadas a manutenção de um ambiente ideal para implementação do cuidado específico a lesão, na intervenção pautada em ações de educação em saúde

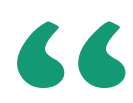

$$
\begin{gathered}
\text {...na utilização } \\
\text { dos insumos e } \\
\text { coberturas de } \\
\text { forma adequada } \\
\text { e sem gerar custos } \\
\text { adicionais ao } \\
\text { sistema de saúde. }
\end{gathered}
$$

no contexto da necessidade específica do usuário, especialmente portador de doenças crônicas, e no desenvolvimento do cuidado baseado na sistemática de acoIhimento e compreensão dos dogmas que interferem negativamente no processo de cicatrização.

Entende-se que há necessidade de maior discussão e avanços científicos no contexto de inserir o enfermeiro enquanto participante ativo do processo de cuidado ao portador de lesões, visto que este profissional possui os atributos necessários para o desenvolvimento de ações de fortalecimento da autonomia e autocuidado garantindo mudanças reais na prevenção e manejo das lesões de pele. Emerge-se ainda, a importância de estudos de avaliação no tocante ao cuidado de enfermagem oferecido a essa população na Atenção Básica em saúde.

\section{Referências}

1. Brito KKG, Sousa MJ, Sousa ATO, Meneses LBA, Oliveira SHS, Soares MJGO, et al. Chronic injuries: nursing approach in the post graduate scientific production. J Nurs UFPE on line [internet]. 2013;7(2):414-21. doi:10.5205/reuol.3073-24791-1LE.0702201312.

2. Conselho Federal de Enfermagem - COFEN. Resolução nº 0567/2018 - Regulamento da atuação da equipe de enfermagem no cuidado aos pacientes com feridas. 3. Chibante CLP, Santo FHE, Santos TD, Porto IS, Daher DV, Brito WAP. Saberes e práticas no cuidado centrado na pessoa com feridas. Esc Anna Nery [internet]. 2017; 21(2): e20170036. doi: https://doi.org/10.5935/1414-8145.20170036

4. Barbiani R, Nora CRD, Schaefer R. Nursing practices in the primary health care context: a scoping review. Rev. Latino-Am. Enfermagem [Internet]. 2016;24:e2721. doi: https://doi.org/10.1590/1518-8345.0880.2721.

5. Jenal S, Vituri DW, Ezaías GM, Silva LA, Caliri MHL. The peer review process: an integrative review of the literature. Acta paul. enferm. [Internet]. 2012; 25(5): 802-808. doi: http://dx.doi.org/10.1590/\$0103-21002012000500024.

6. Melnyk BM, Fineoct-Overholt E. Making the case for evidence-based practice. In: Melnyk BM, Fineoct - Overholt E, editors. Evidence based practice in nursing and healthcare: a guide to best practice. Philadelphia, 2005.

7. Aguiar JS, Brandão ES, Queluci GC, Braga ALS, Soares MF. Physical structure and material resources of the healing halls of regional polyclinics. J Nurs UFPE on line [internet]. 2019;13:e237336. doi: https://doi.org/10.5205/1981-8963.2019.237336. 8. Santos MKS, Martins KP, Santos MCS, Lins WGS, Freitas RSC, Ferreira FA, et al. Nurse orientations to the elderly with diabetes mellitus: preventing injury. J Nurs UFPE online [internet]. 2019;13:e240074. doi: https://doi.org/10.5205/19818963.2019.240074.

9. Chibante CLP, Santo FHE, Santos TD, Porto IS, Daher DV, Brito WAP. Knowledge and practices in care focused on individuals with wounds. Esc. Anna Nery [internet].2017; 21 (2). doi: $10.5935 / 1414-8145.20170036$.

10. Oliveira FP, Oliveira BGRB, Santana RF, SILVA BP, Candido JSC. Nursing interventions and outcomes classifications in patients with wounds: cross-mapping. Revista Gaúcha de Enfermagem [internet]. 2016; 37(2). doi: :http://dx.doi.org/10.1590/19831447.2016.02.55033.

11. Santos El, Oliveira JGAD, Ramos RS, Silva ACSS, Belém LS, Silva AL.Facilities and
Difficulties to the Professional Autonomy of Nurses Caring for People with Wounds: a Study of Social Representations. Revista Estima online [internet]. 2017;15(1). doi: https://doi.org/10.5327/Z1806-3144201700010002.

12. Ministério da Saúde. Manual do Pé Diabético: Estratégias para o cuidado da pessoa com doença crônica. Brasilia/DF. 2016. Disponível em: http://189.28.128.100/ dab/docs/portaldab/publicacoes/manual do pe diabetico.pdf.

13. Gomes RNS, Portela NLC, Pedrosa AO, Monte LRS, Cunha JDS, Soares TR. Evaluation of the physical structure of Basic Health Units. Revista Rene online [internet].2015;16(5). doi: 10.15253/2175-6783.2015000500002.

14. Santos ICRV, Souza MAO, Andrade LNV, Lopes MP, Silva MFAB, Santiago RT. Characterization of care for patients with wounds in Primary Care. Revista Rene online [internet]. 2014; 15(4).doi:10.15253/2175-6783.2014000400008.

15. Colares CMP, Luciano CC, Neves HCC, Tipple AF, Júnior HG.Wound healing and treatment: the interface of knowledge to the practice of the nurse. Enferm. Foco. 2019; 10(3): 52-58. doi: https://doi.org/10.21675/2357-707X.2019.v10.n3.2232. 16. Ribeiro DFS. Care management to chronic wound carriers in Primary Health Care. Revista Enfermagem Atual [internet]. 2019. 90-28. doi: https://doi.org/10.31011/ reaid-2019-v.90-n.28-art.503.

17. Lentsck MH, Baratieri T, Thincaus MR, Mattei AP, Miyahara CTS. Quality of life related to clinical aspects in people with chronic wound. Revista da Escola de Enfermagem da USP [internet]. 2018; 2018;52:e03384. doi:http://dx.doi.org/10.1590/ s1980-220x2017004003384.

18. Alcoforado CLGS, Santo FHE. Knowledge and practices of clients with wounds: a case study in the municipality of Cruzeiro do Sul, Acre. Revista Mineira de Enfermagem[internet]. 2012;16(1): 11-17. Disponivel em:https://cdn.publisher.gn1.link/reme. org.br/pdf/v16n1a02.pdf.

19. Waidman MAP, Rocha SC, Correa JL, BrischiliariA, Marcon SS. Daily routines for individuals with a chronic wound and their mental health. Texto contexto enferm. [online]. 2011; 20(4)691-699. doi: https://doi.org/10.1590/50104-07072011000400007. 20. Busanello J, Silva FM, Sehnem GD, Poll MA, Deus LML, Bohlke TS. Nursing assistance to wounds bearers: technologies of care developed in the primary attention. Revista de Enfermagem da UFSM [internet]. 2013;3(1):175-184. doi:http://dx.doi. org/10.5902/217976928532. 